\title{
COHERENT PION PRODUCTION IN NEUTRINO NUCLEUS COLLISION IN THE 1 GEV REGION
}

\author{
N. G. $\operatorname{Kelkar}^{(1,2)}$, E. Oset ${ }^{(1)}$ and P. Fernández de Córdoba ${ }^{(3)}$ \\ ${ }^{1}$ Departamento de Física Teórica and IFIC, Centro Mixto Universidad de Valencia- \\ CSIC, 46100 Burjassot (Valencia), Spain. \\ 2 Laboratori Nazionali di Frascati, INFN, Italy \\ 3 Departamento de Matemática Aplicada, Universidad Politécnica de Valencia
}

Keywords: Coherent pion production, neutrino nucleus scattering, $\Delta h$ excitation with neutrinos.

PACs numbers: 25.30-c, 25.30. pt

\begin{abstract}
We calculate cross sections for coherent pion production in nuclei induced by neutrinos and antineutrinos of the electron and muon type. The analogies and differences between this process and the related ones of coherent pion production induced by photons, or the $(p, n)$ and $\left({ }^{3} \mathrm{He}, t\right)$ reactions are discussed. The process is one of the several ones occurring for intermediate energy neutrinos, to be considered when detecting atmospheric neutrinos. For this purpose the results shown here can be easily extrapolated to other energies and other nuclei.
\end{abstract}




\section{Introduction}

Electron and muon neutrinos and antineutrinos at intermediate energies are produced in cosmic ray interactions with the earth's atmosphere and they are classified as "atmospheric neutrinos". From basic counting from pion and muon decays one expects twice as many muon neutrinos and antineutrinos as the corresponding electron ones, which seems to be in contradiction with measurements at IMB [1] and Kamiokande [2], where the ratio obtained by studying charged current neutrino nucleus reactions in large underground water detectors is about one. One of the attractive hypothesis is the existence of neutrino oscillations. However, firm conclusions on the reasons of the puzzle can only come if we have a good control on the neutrino nucleus reactions occurring in the detectors, as well as the detector characteristics.

The neutrino nuclear reactions at intermediate energies can be rather complicated if one compares with analogous reactions induced by photons [3]. Most of the studies of neutrino nucleus collisions only consider the ph excitation channel [4, 5, 6]. Others include $\Delta h$ excitation as a source of renormalization of the $p h$ excitation channel but not as an excitation channel by itself [7, 8]. All these approaches are fine in order to evaluate the nucleon emission channel, and at energies below 300-400 MeV they can also provide accurate results for the total neutrino cross section. Indeed, most of the neutrino energy is transferred to the lepton (in charged currents reactions) and only a fraction of it is used to excite the nucleus. Hence, one has to go to relatively large neutrino energies in order to excite $\Delta$ 's and other resonances in the nucleus. Yet, whenever this happens the cross sections for $\Delta h$ excitation are large and comparable in size with those of ph excitation. A recent evaluation of these cross sections is done in ref. [9] and the astrophysical consequences of the consideration of these channels are discussed in ref. [10].

One of the interesting channels discussed in ref. [10 is the coherent pion production following $\Delta$ excitation in the nucleus. The reaction is

$$
\nu_{l}+A \rightarrow l^{-}+A+\pi^{+}
$$

where the final nucleus is the same as the original one and it is left in its ground state. The cross section for the reaction is evaluated in [10] using the impulse approximation (IA), neglecting the $\Delta$ renormalization in the medium and using plane waves for the pion. The authors, however, make a call for accurate calculations which would take those elements into account. Fortunately, such elements have been thoroughly tested in pion nuclear reactions and are readily available. The $\Delta$ selfenergy in a nuclear medium has been evaluated theoretically [11] and tested in all sorts of pion nuclear reactions: elastic [12] absorption, inclusive quasielastic, charge exchange and double charge exchange [13], as well as in pion production processes like coherent $\pi^{0}$ photoproduction 14] or coherent pion production in $\left(p, p^{\prime}\right)$ and related reactions [15].

Coherent pion production in $(p, n)$ or $\left({ }^{3} \mathrm{He}, t\right)$ reactions ressembles much 
the present process and the findings of those reactions can serve as a guideline for the study of the present one. Coherent pion production in the $\left({ }^{3} \mathrm{He}, t\right)$ reaction has received most of the attention [16, 17, 18 since it is one of the channels contributing to the inclusive $\left({ }^{3} \mathrm{He}, t\right)$ reaction in nuclei, where an apparent shift of the $\Delta$ peak with respect to the peak of the elementary reaction on proton targets was observed [19, 20]. In fact the coherent pion production channel shows the peak of the $\Delta$ shifted towards lower excitation energies in all the calculations [16, 17, 18], something already observed in pion elastic scattering as a consequence of the pion multiple scattering [21].

Coherent pion production in neutrino reactions can offer additional information over the $\left(p, p^{\prime}\right),\left({ }^{3} H e, t\right)$ and $(p, n)$ reactions on $\Delta$ properties and pion propagation in the medium. The reason is that in neutrino reactions the range of energy and momentum transferred to the nucleus is different than the one in the hadron induced reactions. On the other hand in the hadronic reactions one has to fight the distortion of the nucleons, or ${ }^{3} \mathrm{He}, t$ in their passage through the nucleus, which makes the pion production reaction quite peripheral. Instead, in the neutrino induced reaction neither the neutrino nor the lepton is distorted by the nucleus and one can test the $\Delta$ and the pions in the interior of the nucleus. One may argue that the same occurs in coherent $\pi^{0}$ photoproduction. However, in this latter case the combination of the spin transverse photons and the spin longitudinal pions leads to a factor $\sin \theta$ in the amplitude which eliminates the contribution of small angles to the cross section. As a consequence the cross section picks up its strength from finite angles where the momentum transfer is larger and hence the nuclear form factor smaller. This reduction due to the form factor becomes more apparent as the energy increases, for a given angle, and as a result of this the $\Delta$ peak is shifted to much smaller energies than in coherent pion production induced by $\left(p, p^{\prime}\right),\left({ }^{3} H e, t\right)$, etc. The contribution of bigger densities is partly to be blamed for the shift, but to a much smaller extent than the reasons discussed above.

In the neutrino induced coherent pion production reaction we do not have the circumstances explained in the photon case and, as we shall see, the largest contribution to the cross section comes from small angles. Then the situation is rather different and we can obtain new information with respect to both the photonuclear and strong interaction induced processes of coherent pion production.

In this sense the neutrino induced coherent pion production reaction is a nice complement to other existing reactions which can enrich our understanding of the nuclear excitation mechanisms at intermedite energies providing new tests for the present theoretical models.

We shall follow the steps and the formalism developed in refs. [15, 17]. Although accurate experimental data on coherent pion production from the $\left(p, p^{\prime}\right)$ or $\left({ }^{3} H e, t\right)$ reactions do not exist, the results of ref. [17] and the preliminary data of ref. [22] for the $\left({ }^{3} \mathrm{He}, t\right)$ induced reaction are in relatively good agreement, which gives us a certain confidence to extend the method to the neutrino induced reaction. 


\section{Coherent pion production amplitude}

The experiments on $\left({ }^{3} \mathrm{He}, t\right)$ induced pion production [19, 20, 22] show that the process is dominated by $\Delta h$ excitation, even at energies of the beam of 10 $\mathrm{GeV}$. The excitation of other resonances is suppressed with respect to the $\Delta$. Only in experiments where the $\Delta$ excitation on the target is forbidden, as in the $\left(\alpha, \alpha^{\prime}\right)$ reaction on a proton target, has the Roper some chances to show up [23], and even then, the Roper signal is small compared to a large background of $\Delta$ excitation in the projectile [23, 24, 25]. The coherent production process still restricts more the excitation of higher resonances since this requires larger momentum transfers which make the nuclear form factor smaller.

The mechanism for coherent pion production in the $\left(\nu, l^{-}\right)$induced reaction proceeds as shown in fig. 1 . The $W^{+}$emitted from the $\left(\nu, l^{-}\right)$vertex excites a $\Delta$ in the nucleus, which decays to $N \pi$ later on, the nucleon remaining in the same original state in order to ensure the coherence.

In order to construct the amplitude for the process we recall the $\nu n \rightarrow l p$ weak interaction Lagrangian

$$
L=\frac{G}{\sqrt{2}} \cos \theta_{c} c^{\mu} J_{\mu}
$$

with

$$
\begin{aligned}
l^{\mu}= & \bar{u}\left(k^{\prime}\right) \gamma^{\mu}\left(1-\gamma_{5}\right) u(k) \\
J_{\mu}= & \bar{u}\left(p^{\prime}\right)\left[F_{1}^{(v)}\left(q^{2}\right) \gamma_{\mu}+\frac{i}{2 M} F_{2}^{(v)}\left(q^{2}\right) \sigma_{\mu \nu} q^{\nu}\right. \\
& \left.+F_{A}^{(v)}\left(q^{2}\right) \gamma_{\mu} \gamma_{5}+F_{P}^{(v)}\left(q^{2}\right) q_{\mu} \gamma_{5}\right] u(p)
\end{aligned}
$$

where $G$ is the Fermi weak coupling constant, $M$ is the nucleon mass and $\theta_{c}$ is the Cabbibo angle. We follow the nomenclature and use the same form factors as in ref. [7]. The momenta involved in eqs. (3) are depicted in fig. 2.

In order to construct the $\nu N \rightarrow l^{-} \Delta$ transition we make the nonrelativistic reduction of the terms in eqs. (3), neglecting only terms of order $O\left(\frac{p}{2 M}\right)^{2}$. Linear terms in $\frac{\vec{p}}{2 M}, \frac{\vec{p}^{\prime}}{2 M}, \frac{\vec{q}}{2 M}$ are kept, but those terms linear in $\vec{p}$, the momentum of the occupied nucleons, also give rise to $O\left(\frac{p}{2 M}\right)^{2}$ corrections when integrating over the momenta. Hence these terms are dropped, which is equivalent to taking $\vec{p}=0, \vec{p}^{\prime}=\vec{q}$. For the $\nu N \rightarrow l^{-} \Delta$ transition we take the terms involving spin operators in the $\nu N \rightarrow l^{-} N$ transition and make the substitution

$$
\frac{f}{\mu} \sigma^{i} \tau^{\lambda} \rightarrow \frac{f^{*}}{\mu} S^{\dagger i} T^{\dagger \lambda}
$$

where $S^{i}, T^{\lambda}$ are the spin, isospin transition operators from $1 / 2$ to $3 / 2$, normalized as

$$
<3 / 2 M_{s}\left|S_{\mu}^{\dagger}\right| 1 / 2 m_{s}>=C\left(1 / 2 \quad 13 / 2 ; m_{s}, \mu, M_{s}\right)
$$


and the same for $T^{\dagger \lambda}$. The couplings $f, f^{*}$ correspond to the $N N \pi$ and $N \Delta \pi$ vertices, $f^{2} / 4 \pi=0.08, f^{* 2} / 4 \pi=0.36$ and $\mu$ in eq. (4) is the pion mass. The current $J_{\mu}$ of eq. (3) contains implicitly a factor $\sqrt{2}$ of the operator $\tau_{+}$ responsible for the $n \rightarrow p$ transition.

Hence we must substitute

$$
\sigma^{i} \rightarrow \frac{f^{*}}{f} \frac{1}{\sqrt{2}} S^{\dagger i} T_{+}^{\dagger}
$$

and from the $\Delta$ decay into $\pi N$ we take $f^{*} / f=2.12$, a value in between the factor 2.2 taken in ref. [26] and the factor 2 considered in ref. [9]

We find

$$
\begin{aligned}
& J_{\mu}^{\Delta}=\frac{1}{\sqrt{2}} \frac{f^{*}}{f}\left\{i\left[F_{1}^{(v)}\left(q^{2}\right)+F_{2}^{(v)}\left(q^{2}\right)\right] \frac{1}{2 M}\left(\vec{S}^{\dagger} \times \vec{q}\right)^{i} \delta_{\mu i}\right. \\
& +\left[F_{A}^{(v)}(q)^{2}-q^{0} F_{P}^{(v)}\left(q^{2}\right)\right] \frac{\vec{S}^{\dagger} \vec{q}}{2 M} \delta_{\mu o}-F_{p}^{(v)}\left(q^{2}\right) \frac{\vec{S}^{\dagger} \vec{q}}{2 M} q^{i} \delta_{\mu i} \\
& \left.+F_{A}^{(v)} S^{\dagger i} \delta_{\mu i}\right\} T_{+}^{\dagger}
\end{aligned}
$$

For the $\Delta$ coupling to a nucleon and a real pion in fig. 1 we use the standard vertex

$$
-i \delta \tilde{H}=-\frac{f^{*}}{\mu} \vec{S} \cdot \vec{p}_{\pi} T^{\lambda}
$$

where $\vec{p}_{\pi}$ is assumed in the $\Delta \mathrm{CM}$ frame. The amplitude corresponding to fig. 1 is readily evaluated for a spin saturated nucleus using the property

$$
\sum_{M_{s}} S_{i} S_{j}^{\dagger}=\frac{2}{3} \delta_{i j}-\frac{i}{3} \epsilon_{i j l} \sigma_{l}
$$

One of the findings in coherent pion production induced by the $\left({ }^{3} \mathrm{He}, t\right)$ reaction was the negligible contribution from the transverse part of the $N N \rightarrow$ $N \Delta$ transition amplitude. This occurred because the emitted pion couples longitudinally to the $\Delta$ and the transverse part of the interaction contributes to the cross section with the factor $\sin ^{2} \theta$, as we indicated for the case of coherent pion photoproduction. This forces the contribution at finite angles where the nuclear form factor reduces the cross section. This is also the case here, where in addition the transverse terms are further reduced by a factor $q / 2 M$. Hence we neglect the transverse parts from the beginning.

With all these considerations, the coherent pion production cross section corresponding to the process of fig. 1, on summing over all occupied nucleons in the amplitude, is given by

$$
\frac{d \sigma}{d \Omega_{e} d E_{e} d \Omega_{\pi}}=\frac{1}{8} \frac{\left|\vec{k}^{\prime}\right|\left|\vec{p}_{\pi}\right|}{|\vec{k}|} \frac{1}{(2 \pi)^{5}} \Pi_{f} 2 m_{f} \bar{\Sigma} \Sigma|t|^{2}
$$

where the product of the fermion masses, $2 m_{f}$, appears because of our normalization of the spinors as $\bar{u} u=1$. The $T$ matrix squared, summed and averaged over spins, is given by 


$$
\Pi_{f} 2 m_{f} \bar{\Sigma} \Sigma|t|^{2}=L_{00}\left|V^{0}\right|^{2}+L_{33}\left|V^{3}\right|^{2}+2 L_{03} \operatorname{Re}\left\{V^{0} V^{3 *}\right\}
$$

where $L_{\mu \nu}$ is the leptonic tensor

$$
L_{\mu \nu}=4\left[k_{\mu} k_{\nu}^{\prime}+k_{\mu}^{\prime} k_{\nu}-k \cdot k^{\prime} g_{\mu \nu}\right]
$$

and

$$
\begin{gathered}
V^{\mu} \equiv\left\{\begin{array}{c}
V^{0} \\
0 \\
0 \\
V^{3}
\end{array}\right\} \\
V^{0}=B\left[F_{A}^{(v)}\left(q^{2}\right)-q^{0} F_{P}^{(v)}\left(q^{2}\right)\right] \\
V^{3}=B\left[F_{A}^{(v)}\left(q^{2}\right) \frac{2 M q}{\vec{q}^{2}}-F_{P}^{(v)}\left(q^{2}\right) q\right]
\end{gathered}
$$

with

$$
B=-\frac{f^{*}}{f} \frac{f^{*}}{\mu} \frac{G}{6} \cos \theta_{c} G_{\Delta}\left(p_{\Delta}\right) F\left(\vec{q}-\vec{p}_{\pi}\right) \frac{1}{\sqrt{s_{\Delta}}}
$$

In the factor $B, G_{\Delta}\left(p_{\Delta}\right)$ is the $\Delta$ propagator and $F\left(\vec{q}-\vec{p}_{\pi}\right)$ is the nuclear form factor modulated by the isospin factors. We have

$$
G_{\Delta}\left(p_{\Delta}\right)=\frac{1}{\sqrt{s_{\Delta}}-M_{\Delta}+i \frac{\tilde{\Gamma}}{2}-\Sigma_{\Delta}}
$$

where $s_{\Delta}=p_{\Delta}^{02}-\vec{p}_{\Delta}^{2}$, and $\tilde{\Gamma}, \Sigma_{\Delta}$ are the Pauli blocked $\Delta$ width and the rest of the $\Delta$ selfenergy which contains pieces related to quasielastic scattering, $2 N$ and $3 N$ pion absorption. The evaluations are done in ref. 11] and we take the analytic expressions derived there. We also include in the selfenergy the term $\frac{4}{9}\left(\frac{f^{*}}{\mu}\right)^{2} g^{\prime} \rho$ to account for irreducible pieces of $\Delta h$ propagation mediated by the Landau-Migdal effective interaction [12].

On the other hand the nuclear form factor is given by

$$
F\left(\vec{q}-\vec{p}_{\pi}\right)=\int d^{3} r\left[\rho_{p}(\vec{r})+\frac{1}{3} \rho_{n}(\vec{r})\right] e^{i \vec{q} \cdot \vec{r}} \vec{p}_{\pi} \cdot \vec{q} e^{-i \vec{p}_{\pi} \cdot \vec{r}}
$$

where for convenience we have included the factor $\vec{p}_{\pi} \cdot \vec{q}$.

In the derivation of eq. (11) we have taken $\vec{q}$ in the $\mathrm{z}$ direction for simplicity and furthermore we have also kept only the longitudinal part of $\vec{p}_{\pi}$ along the $q$ axis for consistency with the neglect of the transverse parts. The structure of eq. (11) is also the same as the one found in ref. [10].

It is interesting to note that when $\vec{k}^{\prime}$ is paralel to $\vec{k}$, which leads to the largest cross sections, the contribution of $F_{P}$ cancels. Hence, the axial term $F_{A}^{(v)}\left(q^{2}\right)$ is the relevant term in the process.

So far the formalism has used the bound wave functions of the nucleus, which appear in the nuclear form factor via the proton and neutron densities, 
eq. (16), but has considered only a plane wave for the pion. The renormalization of the pion is a very important thing in this process. Hence, in the next step we replace

$$
\vec{p}_{\pi} \cdot \vec{q} e^{-i \vec{p}_{\pi} \cdot \vec{r}} \rightarrow i \vec{q} \cdot \vec{\nabla} \phi_{\text {out }}^{*}\left(\vec{p}_{\pi}, \vec{r}\right)
$$

where $\phi_{\text {out }}^{*}\left(\vec{p}_{\pi}, \vec{r}\right)$ is an outgoing solution of the Klein Gordon equation for the pion, which we solve along the lines of ref. [27] and with the pion nucleus optical potential developed there, which gives rise to good pion elastic, absorption and quasielastic cross sections [27].

\section{Results and discussion}

In fig. 3 we plot $d \sigma / d \Omega_{e} d E_{e} d \Omega_{\pi}$, for $\theta_{e}=0^{0}$ with respect to the neutrino direction, for coherent pion production in $\nu_{e}+{ }^{16} O \rightarrow e^{-}+{ }^{16} O+\pi^{+}$as a function of the pion angle measured with respect to $\vec{q}$. We choose a neutrino energy of $800 \mathrm{MeV}$ and an electron energy of $545 \mathrm{MeV}$ which lead to a value of $q$ suited to excite the $\Delta$ resonance. The dashed line corresponds to the impulse approximation, meaning free $\Delta$ width and no $\Delta$ selfenergy in the $\Delta$ propagator and no pion distortion. The solid line is the accurate calculation, which accounts for both effects. We can see that there is a net reduction of about a factor three from both renormalizations, bigger than what appears in coherent pion production induced by the $\left({ }^{3} \mathrm{He}, t\right)$ reaction, which is more peripheral.

The cross section is forward peaked, as was also the case in the $\left({ }^{3} \mathrm{He}, t\right)$ or $(p, n)$ reactions. However the fall down with angle is not so drastic here as in the hadronic reactions because in the latter ones, for the same energy of the pion, the momentum transferred to the nucleus is bigger than in the neutrino case, as a consequence of the large mass of the nucleons, and the nuclear form factor reduces more the cross section. The cross sections are of the order of $10^{-15} \mathrm{fm}^{2} / \mathrm{MeVsr}$.

In fig. 4 we show the cross section for the same reaction integrated over the pion angles. Here we plot it as a function of $q^{0}$, the total pion energy (we neglect the nucleus recoil energy). We observe again the sizeable renormalization factor from dressing the $\Delta$ and the pion in the nuclear medium. It is also worth looking at the shift to lower energies of the peak of the excitation function, with respect to the one of the impulse approximation. This is mostly due to the distortion of the pion, as we already indicated in the introduction. The argument goes as follows: since the $\pi N$ cross section and pion absorption are largest at resonance, there is a depletion of the pion wave when the pion goes through the nucleus, and much of the pion flux is lost into quasielastic channels or pion absorption. On the other hand the pion production step is resonance peaked. The combination of these two factors has as a consequence a shift of the peak to lower excitation energies where the pion depletion is not so strong. Note, however, that the free $\Delta$ position would appear at $q^{0}=338 \mathrm{MeV}$ in the 
plot. Hence, we see a shift of the peak already in the IA due to the nuclear form factor, as indicated in the introduction, and a further shift due to the pion distortion.

The nuclear form factor acts as follows: from energy conservation we have $\omega_{\pi}=q^{0}, p_{\pi}=\left(q^{02}-\mu^{2}\right)^{1 / 2}$. Hence, the momentum transfer to the nucleus, $\vec{q}-\vec{p}_{\pi}$, is always finite since $q>q^{0}$ and it increases as $q^{0}$ increases. As a consequence, the nuclear form factor decreases with increasing $q^{0}$ and this has the same effect as the distortion when one approaches the $\Delta$ energy, leading to a shift of the $\Delta$ peak to lower excitation energies.

The $\Delta$ peak in fig. 4 appears around $q^{0}=255 \mathrm{MeV}$, which is lower than the value $275 \mathrm{MeV}$ found in coherent $\pi^{+}$production with the $(p, n)$ reaction. Once again the peripheral character of the $(p, n)$ reaction should be blamed for it. However, it is interesting to note that, in spite of the fact that here we are exploring the whole volume of the nucleus, like in coherent $\pi^{0}$ photoproduction, the peak appears at higher values of $q^{0}$ in the neutrino case than in the $\left(\gamma, \pi^{0}\right)$ [14, 28] case. In the latter case the $\Delta$ peak was shifted to energies around $190-220 \mathrm{MeV}$ depending on the nucleus. The reason, already discussed in the introduction, is the factor $\sin ^{2} \theta$ of coherent $\pi^{0}$ photoproduction which forces finite angles in the cross section where the momentum transfer is larger and the nuclear form factor smaller.

As we can see, the combination of results of coherent pion production induced by photons, neutrinos or hadronic reactions presents complementary aspects related to the nuclear properties and the propagation of the $\Delta$ and the pion in the nuclear medium.

In fig. 5 we show the results for the cross section of the same reaction integrated now over the electron angles. We observe similar features as in fig. 4. The magnitude of the cross section has now decreased more with respect to the case where we integrate over the pion angles (see figs. 3 and 4 ), indicating that the cross section as a function of the electron angle is more forward peaked than with the pion angle. This is intuitive since the electron momentum is bigger than the pion momentum and a change in angle generates larger momentum transfers in the case of the electron, which would lead to a larger reduction of the nuclear form factor.

In fig. 6 we show the results for $d \sigma / d E_{e}$ as a function of $q^{0}$ for different neutrino energies. We can see that the cross section increases with the neutrino energy, but at energies above $1 G e V$ the increase is more moderate. This is also reminiscent of the findings of ref. [15] in the $(p, n)$ reaction.

In fig. 7 we show the results of $d \sigma / d E_{e}$ as a function of $q^{0}$, for neutrinos of $1 \mathrm{GeV}$ scattering from three different nuclei, ${ }^{16} \mathrm{O},{ }^{37} \mathrm{Cl}$ and ${ }^{71} \mathrm{Ga}$ used as neutrino detectors in several experiments. We observe that the cross section grows with $\mathrm{A}$. This is quite different from the results found for the $(p, n)$ reaction where the cross section decreased from ${ }^{12} \mathrm{C}$ to ${ }^{40} \mathrm{Ca}$ and ${ }^{208} \mathrm{~Pb}$. The reason for the decrease in the hadronic reaction was the distortion of the $p$ and $n$ waves, which does not occur now, since the neutrino and the electron are not distorted by the nucleus. Finally in fig. 8 we show the results for the 
reaction

$$
\nu_{\mu}+A \rightarrow \mu^{-}+A+\pi^{+}
$$

corresponding to fig. 7 with electrons.

The evaluation of the cross section in the $\nu_{\mu}$ case is simple since both the leptonic tensor and the vector $V^{\mu}$ have the same expressions. The only change is in the kinematics in the $\left(\nu_{\mu}, \mu\right)$ vertex because of the finite mass of the muon. The cross sections for the $\nu_{\mu}$ case are decreased by about $20 \%$ with respect to those of $\nu_{e}$ in all nuclei. The reason for the decrease is that for a given value of $q^{0}$ the corresponding value of $q$ is larger in the $\mu$ case and hence it leads to larger momentum transfers to the nucleus and smaller nuclear form factors.

Since the Laboratory energetic neutrinos are muon neutrinos, the reaction studied here could be implemented with muon neutrinos in present Laboratories.

As for the evaluation of cross sections with antineutrinos, the changes to be done to obtain them from the ones evaluated in this work are minimal, once the transverse parts are neglected as done here. One should change

$$
\rho_{p}+\frac{1}{3} \rho_{n} \rightarrow \rho_{n}+\frac{1}{3} \rho_{p}
$$

which in practice amounts to multiplying the neutrino cross sections by the factor $(3 N+Z) /(3 Z+N)$, which is unity for isospin symmetric nuclei, as noted in ref. [10].

\section{Conclusions}

We have calculated cross sections for coherent pion production in neutrino (antineutrino) nucleus collisions, of both electron and muon type. The calculations have been done accurately taking into account the renormalization of the $\Delta$ and pion properties in the nuclear medium. We observed that the cross section was quite sensitive to these properties, and their inclusion in the calculation decreased the cross section by about a factor three with respect to the impulse approximation, and shifted the peak position to lower excitation energies.

Some of the features, like the shift of the $\Delta$ peak, were reminiscent of similar findings in coherent pion production in $\left({ }^{3} \mathrm{He}, t\right)$ or $(p, n)$ reactions, but the fact that the latter are rather peripheral because of the distortion of the hadronic beam, by contrast to the neutrino reaction which occur throughout the nuclear volume, confers the neutrino reaction some peculiar features. These features

are also different to those found in coherent $\pi^{0}$ photoproduction, also testing the whole nuclear volume, because in the latter case there is a factor $\sin ^{2} \theta$ in the cross section which reduces the contribution of forward angles from where the neutrino cross sections get most of their contribution. 
All these analogies and differences tell us that the coherent pion production induced by neutrinos is an important complement of the hadronic and photonuclear processes of pion production in order to give information on pion and $\Delta$ renormalization in a nuclear medium.

On the other hand it is clear that in order to obtain proper information about atmospheric neutrinos one has to have a control on the different $\nu$ nuclear reactions occuring at intermediate energies of the neutrinos, to interpret properly the results of the neutrino detectors. The present reaction is one of them.

In order to test the validity of the model used to obtain the present results, which can be easily extrapolated to other nuclei and other energies, it would be interesting to perform some experiment. The cross sections, although small, are in the same range as in many experiments performed at present facilities 29] and hence are experimentally accessible.

\section{Acknowledgements:}

We would like to acknowledge partial support from CICYT contract no. AEN 96- 1719. One of us, N. G. K. wishes to acknowledge the hospitality of the Valencia University where this work has been done. 


\section{References}

[1] R. Becker-Szendy et al., Phys. Rev. D 46, 3720 (1992).

[2] K. S. Hirata et al., Phys. Lett. 280B, 146 (1992); E. W. Beier et al., Phys. Lett. 283B, 446 (1992).

[3] R. C. Carrasco and E. Oset, Nucl. Phys. A536, 445 (1992).

[4] W.C. Haxton, Phys. Rev. D 36, 2283 (1987).

[5] K. Kubodera and S. Nozawa, Int. J. Mod. Phys. E3, 101 (1994).

[6] J. Engel, S. Pittel and P. Vogel, Phys. Rev. Lett. 67, 426 (1991); Phys. Rev. C 50, 1702 (1994).

[7] S. K. Singh and E. Oset, Nucl. Phys. A542, 587 (1992); Phys. Rev. C 48, 1246 (1993).

[8] T. S. Kosmas and E. Oset, Phys. Rev. C 53, 1409 (1996).

[9] H. Kim, S. Schramm and C. J. Horowitz, Phys. Rev. C 53, 2468 (1996).

[10] H. Kim, S. Schramm and C. J. Horowitz, Indiana preprint IU/NTC 96-02.

[11] E. Oset and L.L. Salcedo, Nucl. Phys. A468, 631 (1987).

[12] C. García-Recio, E. Oset, L.L. Salcedo, D. Strottman and M.J. López, Nucl. Phys. A526, 685 (1991).

[13] L.L. Salcedo, E. Oset, M.J. Vicente Vacas and C. García Recio, Nucl. Phys. A484, 557 (1988).

[14] R.C. Carrasco, J. Nieves and E. Oset, Nucl. Phys. A565, 797 (1993).

[15] P. Fernández de Córdoba, E. Oset and M.J. Vicente-Vacas, Nucl. Phys. A592, 472 (1995).

[16] P. Oltmanns, F. Osterfeld and T. Udagawa, Phys. Lett. 299B, 194 (1993).

[17] P. Fernández de Córdoba, J. Nieves, E. Oset and M.J. Vicente-Vacas, Phys. Lett 319B, 416 (1993).

[18] V. F. Dmitriev, Phys. Rev. C 48, 357 (1993).

[19] C. Ellegaard et al., Phys. Rev. Lett. 50, 1795 (1983).

[20] V. G. Ableev et al., Sov. Phys. JEPT Lett. 40, 763 (1984).

[21] T.E.O. Ericson and W. Weise, Pions and Nuclei, Clarendon, Oxford, 1988.

[22] T. Hennino et al., Phys. Lett. 303B, 236 (1993). 
[23] H.P. Morsch et al., Phys. Rev. Lett. 69, 1336 (1992).

[24] P. Fernández de Córdoba, E. Oset, M.J. Vicente-Vacas, Yu. Ratis, J. Nieves, B. López-Alvaredo and F. Gareev, Nucl. Phys. A586, 586 (1995).

[25] S. Hirenzaki, P. Fernández de Córdoba and E. Oset, Phys. Rev. C 53, 277 (1996).

[26] M.J. Dekker, P.J. Brussard and J.A. Tjon, Phys. Rev. C 49, 2650 (1994).

[27] J. Nieves, E. Oset and C. García-Recio, Nucl. Phys. A554, 554 (1993).

[28] I. Laktineh, W.M. Alberico. J. Delorme and M. Ericson, Nucl. Phys. A555, 237 (1993).

[29] B. Zeitnitz, Prog. Part. Nucl. Phys. 32, 351 (1994). 


\section{Figure Captions}

1. Diagrammatic representation of the coherent pion production process $\nu_{e}+A(g . s) \rightarrow e^{-}+A($ g.s. $)+\pi^{+}$.

2. Kinematics of the $\nu n \rightarrow e^{-} p$ process.

3. Angular distribution of the pions from coherent $\pi^{+}$production on ${ }^{16} \mathrm{O}$ with neutrino beam of energy $800 \mathrm{MeV}, T_{e}=545 \mathrm{MeV}$ and $\theta_{e}=0^{0}$. The solid curve corresponds to the full calculation which includes renormalization of the $\Delta$ and pion in the nuclear medium. The dashed curve is the impulse approximation calculation.

4. Energy spectrum of the coherent pions produced on ${ }^{16} \mathrm{O}$ with neutrinos of beam energy $1 \mathrm{GeV}$, and $\theta_{e}=0^{0}$. The solid curve corresponds to the full calculation with renormalized pions and deltas and dashed line is the impulse approximation calculation.

5. Same as fig.4, but integrated over the electron solid angle.

6. Energy spectra of the coherent pions produced on ${ }^{16} O$, at three different neutrino energies, with the $\Delta$ and pion renormalizations included in the calculations.

7. Energy spectra of the coherent pions scattered from three different nuclei by neutrinos of $1 \mathrm{GeV}$ energy.

8. Energy spectra of the coherent pions scattered from three different nuclei by muon type neutrinos of $1 \mathrm{GeV}$ energy. 